\section{Estudo da validação das informações de peso e estatura em gestantes atendidas em maternidades municipais no Rio de Janeiro, Brasil}

\author{
Study of validity in self-reported weight and height \\ among pregnant women treated at municipal \\ maternity hospitals in Rio de Janeiro, Brazil
}

\author{
1 Departamento de \\ Epidemiologia e Métodos \\ Quantitativos em Saúde, \\ Escola Nacional de Saúde \\ Publica, Fundação Oswaldo \\ Cruz, Rio de Janeiro, Brasil. \\ 2 Departamento de \\ Informações em Saúde, \\ Centro de Informação \\ Científica e Tecnológica, \\ Fundação Oswaldo Cruz, \\ Rio de Janeiro, Brasil. \\ Correspondência \\ Andreia Ferreira de Oliveira \\ Rua Leopoldo Bulhões 1480, \\ Rio de Janeiro, $R$ \\ 21041-210, Brasil. \\ andreiaf@ensp.fiocruz.br
}

\begin{abstract}
The main objectives of this article are the assessment of agreement between measure-based and report-based information on weight and height, besides the identification of the main characteristics related to 150 pregnant women who could report (versus could not report) their pre-gestational weight, weight at delivery, and height. According to the results, report-based information on previous weight is close to the measure-based information (Pearson coefficient correlation 0.96 and intra-class correlation 0.92; $p<0.05$ ), with the same occurring for weight at delivery (Pearson coefficient correlation 0.96 and intraclass correlation 0.95; $p<0.05)$. Nevertheless, linear regression analysis detects that this relationship is troublesome for the height variable $\left(\beta_{0}=45.5\right.$ and $\left.\beta_{1}=0.71 ; p<0.05\right)$, and therefore the report-based information for height must be used cautiously when the measure-based values are not available. In relation to the characterization of the group of pregnant women who did not inform the three measures $(n=141)$, these women have the least schooling (63.9\%), did not attend or attended fewer prenatal appointments (64.3\%), and entered the prenatal program later, when they were already in the second or third trimester (59.3\%).
\end{abstract}

Validity; Body Weight; Body Height; Pregnancy
Andreia Ferreira de Oliveira 1 Angela Maria Jourdan Gadelha 1 Maria do Carmo Leal 1 Célia Landmann Szwarcwald 2

\section{Introdução}

O monitoramento nutricional na gestação, com seu impacto positivo na saúde materno-fetal e no pós-parto, tem sido apontado como elemento fundamental na prevenção da morbi-mortalidade perinatal, prognóstico da situação de saúde da criança nos primeiros anos de vida e na promoção da saúde da mulher 1 .

Entre os métodos de avaliação utilizados, as medidas antropométricas de peso e estatura são habitualmente empregadas. No entanto, quando essas medidas não são diretamente aferidas, torna-se necessário empregarem-se valores referidos pela própria gestante. A validade das informações de peso e estatura em gestantes pode fornecer subsídios para sua utilização em substituição aos valores medidos, sendo particularmente relevante para o acompanhamento do ganho de peso na gestação 2 .

Considerando que as mulheres não ganham peso e, até mesmo, perdem em média $0,2 \mathrm{~kg} \mathrm{du}$ rante o primeiro trimestre de gestação, o peso tomado neste período tem sido utilizado como proxis do peso pré-gestacional $3,4,5$. O peso prégestacional é de grande importância no cálculo do índice de massa corporal (IMC) pré-gestacional, o qual é utilizado na avaliação do ganho de peso total durante a gestação, segundo estado nutricional materno prévio e sua influência na saúde materna e fetal 2. 
Vários estudos têm se dedicado a investigar a validade das informações referidas de peso e estatura em adultos. Os resultados encontrados em estudos internacionais evidenciaram diferenças estatisticamente significativas entre os valores medidos e os referidos, com tendência à subestimação dos valores de peso e superestimação dos valores para estatura referidos 6,7,8,9.

No Brasil, foram encontrados dois estudos voltados à validação das informações de peso e estatura. $\mathrm{O}$ trabalho realizado por Schmidt et al. 10 avaliou a validade das informações de peso referidas em 659 adultos de 15-64 anos em Porto Alegre, Rio Grande do Sul, verificando-se elevada correlação $(r=0,97)$ entre as informações de peso medido e referido, com tendência à superestimação entre os indivíduos classificados como baixo peso e subestimação entre os obesos. Já a pesquisa realizada por Chór et al. 11, em amostra aleatória simples de 1.183 funcionários de uma empresa, evidenciou que homens e mulheres tenderam a subestimar seu peso, não se observando diferenças quanto à estatura.

$\mathrm{Na}$ literatura científica internacional, alguns trabalhos abordavam notadamente a população de gestantes 12,13,14,15,16. Não foram encontradas pesquisas brasileiras neste grupo populacional.

Como justificativa para a realização deste trabalho, ressalta-se a importância das informações referidas como elemento facilitador no monitoramento do estado nutricional durante a gestação para os serviços e/ou assistência pré-natal.

Os principais objetivos deste estudo foram avaliar a concordância entre peso e estatura referidos com a medida direta através da utilização de diferentes métodos de análise, bem como identificar as características das gestantes que soubessem referir seu peso anterior, peso final e estatura em face daquelas que não souberam.

\section{Metodologia}

Esta pesquisa se constitui em um subprojeto do "Estudo da Morbi-mortalidade e da Atenção Peri e Neonatal no Município do Rio de Janeiro", desenvolvido com base em uma amostra de puérperas que se hospitalizaram em maternidades do município por ocasião do trabalho de parto, no período de julho 1999 a março de 2001. Fizeram parte desta pesquisa as seguintes instituições: Fundação Oswaldo Cruz (FIO-
CRUZ), Secretaria Municipal de Saúde (SMSRJ) e Instituto de Pesquisa Econômica e Aplicada (IPEA), sendo financiada com recursos da Fundação Carlos Chagas Filho de Amparo à Pesquisa do Estado do Rio de Janeiro (FAPERJ). A metodologia deste trabalho foi descrita com maior detalhamento por Leal et al. 17 .

O cálculo do tamanho amostral foi baseado no teste $t$ de hipóteses para comparação de duas médias com dados emparelhados, pressupondo a normalidade da distribuição amostral da variável teste 18 . Como não havia referência bibliográfica que estabelecesse aproximações ao desvio padrão para ser utilizado no cálculo das diferenças médias de peso e estatura em populações de gestantes no cálculo do tamanho amostral, foram utilizados os dados do estudo-piloto desenvolvido no Hospital Geral de Bonsucesso, onde cerca de 86 entrevistas foram realizadas.

A amostra foi selecionada da população de gestantes que deu entrada nos Hospitais Estaduais Rocha Faria e Pedro II por ocasião do trabalho de parto. Foram realizadas 291 entrevistas, incluindo-se na amostra aquelas com feto único e que se encontravam a partir da trigésima sétima semana gestacional. Destas, apenas 150 referiram as informações do peso anterior, peso final e estatura, sendo o total utilizado para fins de análise.

As variáveis do estudo foram: peso e estatura medidos e referidos, idade, grau de instrução, situação conjugal, número de partos, mês de início, número de consultas e freqüência de pesagem durante o pré-natal. O peso pré-gestacional medido foi obtido do cartão pré-natal, sendo as demais informações extraídas do questionário da pesquisa.

A coleta dos dados de peso e estatura foi realizada pela própria autora deste trabalho, que foi treinada no Ambulatório de Nutrição da Policlínica Piquet Carneiro, Universidade do Estado do Rio de Janeiro, por dois dias consecutivos. O objetivo foi a padronização na tomada das medidas de peso e estatura segundo critérios propostos por Lhoman 19.

O peso e estatura maternos foram mensurados pela pesquisadora na sala do pré-parto, sendo os dados registrados em um questionário elaborado para este fim. Como instrumento para coleta de informações sobre peso, utilizouse uma balança eletrônica portátil (UNICEF) com capacidade de $150 \mathrm{~kg}$ e precisão de $100 \mathrm{~g}$, em local firme e superfície plana. Para tomada da estatura, empregou-se uma fita metálica afi- 
xada em superfície vertical, sem rodapés, com auxílio de fitas adesivas, registrando-se a estatura em centímetros. Para a medição do peso em quilogramas, as gestantes removeram os sapatos e utilizaram roupas leves (fornecidas pela própria maternidade).

Por meio do teste $t$ de Student, analisaramse as diferenças entre as médias de peso anterior e final da gestação e de estatura medida e referida. Essa análise para o peso pré-gestacional foi realizada apenas para as gestantes que iniciaram o pré-natal no primeiro trimestre e tinham a informação do peso anterior no cartão de pré-natal, totalizando trinta gestantes. Para comparação das distribuições segundo quartis do peso pré-gestacional medido e referido, utilizou-se o teste de Kruskal-Wallis, ao passo que a análise para o peso final e estatura medidos e referidos foi feita utilizando-se o teste $\chi^{2}$. Com os dados de peso final e estatura referidos distribuídos por quartis, analisou-se a sensibilidade tomando-se como valor padrão a informação medida, aplicando-se o coeficiente Kappa para verificar o grau de concordância. Os critérios utilizados para avaliação dos valores de Kappa foram os propostos por Fleiss 20: concordância excelente $(k>0,75)$, boa $(0,4<\mathrm{k}<0,75)$ e pobre $(\mathrm{k}<0,4)$. Em virtude do pequeno tamanho amostral $(n=30)$, não foram calculados os valores da sensibilidade para a variável peso anterior.

Para estabelecer um critério de análise de discordância, utilizou-se o método gráfico proposto por Bland \& Altman 21, construindo-se histogramas da distribuição de freqüências das diferenças de peso anterior, peso final e estatura medidos e referidos em função da média desses valores.

Calculou-se o coeficiente de correlação de Pearson e de correlação intraclasse (CCIC) entre as variáveis de peso anterior, peso final e estatura medidos e referidos. Seus escores variam de $-1 \mathrm{a}+1$, com os mais altos valores refletindo um aumento na concordância observada 21 . O uso do CCIC permitiu avaliar a concordância entre as informações, uma vez que o coeficiente de correlação de Pearson avalia somente relações lineares entre as variáveis e não leva em consideração o viés sistemático 21,22.

Por meio de análise de regressão linear, testou-se a hipótese de que os coeficientes de regressão $\beta_{0}$ e $\beta_{1}$ corresponderiam a zero e a um, respectivamente, utilizando como variáveisresposta os valores medidos e como variáveis independentes os valores referidos.

Para comparação da proporção de gestantes que sabiam, ou não, referir seu peso anterior, peso final e estatura segundo característi- cas selecionadas, realizou-se análise comparativa de distribuição de freqüência para as variáveis independentes entre os grupos de gestantes que sabiam ou não referir pelo menos uma das três informações.

\section{Resultados}

\section{Validação da medida de peso final e estatura}

A média das diferenças entre peso final e estatura medidos e referidos foram de $0,5 \mathrm{~kg} \mathrm{e}-1,23 \mathrm{~cm}$, respectivamente, indicando a tendência, por parte dessas gestantes, de subestimarem o peso e superestimarem a estatura (Tabela 1). A análise das diferenças médias de peso final e estatura segundo os percentis dos valores medidos mostrou que não existem diferenças estatisticamente significantes $(p>0,05)$ entre as classes estabelecidas. A distribuição de freqüência do peso e estatura medidos e referidos segundo os quartis correspondentes evidenciou que os valores da sensibilidade foram mais elevados nos primeiros $(94,6 \%$ para peso e $83,8 \%$ para estatura) e últimos quartis $(91,9 \%$ para peso e $75,7 \%$ para estatura) em comparação às demais categorias, indicando que aquelas 'mais baixas e magras' e as 'mais altas e pesadas' apresentaram melhor concordância ao se comparar a informação referida com a medida. O coeficiente de Kappa (k) encontrado para o peso final ( $\mathrm{k}=0,84$; IC95\%: 0,77-0,90) e estatura ( $k=0,55$; IC95\%: 0,45-0,65) apresentaram $\mathrm{p}$-valor significativamente diferente de zero $(\mathrm{p}=0,000)$.

Na análise de regressão linear para o peso final, primeiramente foram testados modelos incluindo os valores outliers da distribuição, evidenciando resultados significativos para os coeficientes linear e angular $(\mathrm{p}<0,05)$. Com isso, optou-se por realizar análise excluindo os valores discrepantes (considerados como aqueles cujo valor do resíduo padronizado fosse maior que dois), cujos valores dos coeficientes linear e angular $(\mathrm{p}>0,05)$ não foram significativos, indicando que a hipótese de $\beta_{0}=0$ e $\beta_{1}=1$ foi aceita.

Para a estatura, foi realizada análise semelhante à do peso final. Primeiramente testouse um modelo que também incluiu os valores outliers da distribuição, em que os coeficientes linear e angular apresentaram significância estatística $(\mathrm{p}<0,05)$. Em virtude de tal fato, optou-se pela retirada das observações discrepantes. Os resultados evidenciaram que os parâmetros $\beta_{0}(\mathrm{p}<0,05)$ e $\beta_{1}(\mathrm{p}<0,05)$ ainda assim mantiveram significância estatística, indi- 
cando que, para a variável estatura, as informações referidas pelas gestantes necessitariam de ajuste por meio da equação de regressão (Tabela 1).

Utilizando a metodologia proposta por Bland \& Altman 21, construíram-se os gráficos das diferenças entre peso final e estatura medidos e referidos em função da média desses valores (Figuras 1 e 2). A maior concentração de pontos positivos acima da linha de referência para o peso e abaixo para a estatura indicaram que as gestantes subestimaram o peso e superestimaram a estatura, respectivamente.

O coeficiente de correlação de Pearson, utilizado para avaliar a correlação entre as informações medidas e referidas de peso final e estatura, apresentou valores de 0,96 (p<0,05) para peso e $0,82(\mathrm{p}<0,05)$ para estatura, indicando uma boa correlação entre as variáveis. A concordância entre as informações foi avaliada por meio do coeficiente de correlação intraclasse (CCIC), cujos valores encontrados foram de 0,95 ( $\mathrm{p}<0,05)$ para o peso (IC95\%: 0,940,97 ) e $0,79(\mathrm{p}<0,05)$ para estatura (IC95\%: 0,72-0,85).

\section{Validação da medida de peso anterior}

A média das diferenças foi de $2,7 \mathrm{~kg}$, indicando que as gestantes subestimam seu peso pré-gestacional (Tabela 1). Na comparação das diferenças médias de peso anterior segundo as classes de peso estabelecidas por meio de quartis, utilizou-se o teste de Kruskal-Wallis e os resultados não apresentaram significância estatística no nível de 5\%, indicando que não existem diferenças marcantes entre as categorias.

A Figura 3 apresenta as diferenças entre o peso pré-gestacional medido e o referido em função da média destes valores, segundo a metodologia proposta por Bland \& Altman 21. Os resultados apresentaram maior distanciamento dos pontos em relação à linha média da diferença $(2,7 \mathrm{~kg})$. Verificam-se menores diferenças médias entre aquelas que possuíam média de peso abaixo de $60 \mathrm{~kg}$ e maiores diferenças entre as que se apresentavam acima desse ponto. Todos os valores encontrados para a média das diferenças são positivos, indicando tendência, por parte dessas gestantes, a subestimarem seus pesos anteriores.

A correlação e concordância entre o peso anterior medido e o referido foram elevadas, com valores do coeficiente de correlação de Pearson e intraclasse de 0,96 (p<0,05) e 0,92 ( $p<$ 0,05; IC95\%: 0,52-0,97), respectivamente.

$\mathrm{Na}$ análise de regressão linear não foi excluído nenhum valor discrepante da distribui-
Tabela 1

Principais resultados quanto aos métodos empregados na validação das informações de peso anterior, final e estatura em gestantes no Município do Rio de Janeiro, Brasil.

\begin{tabular}{|c|c|c|c|}
\hline Métodos aplicados & $\begin{array}{c}\text { Peso anterior } \\
(\mathrm{kg})\end{array}$ & $\begin{array}{c}\text { Peso final } \\
(\mathrm{kg})\end{array}$ & $\begin{array}{l}\text { Estatura } \\
\text { (cm) }\end{array}$ \\
\hline Média das diferenças & 2,70 & 0,50 & $-1,23$ \\
\hline \multicolumn{4}{|l|}{ Diferenças por quartis } \\
\hline $1 \underline{0}$ & 0,70 & 0,17 & 0,31 \\
\hline 2으 & 4,22 & 0,21 & 2,52 \\
\hline 3으 & 2,32 & 1,16 & 1,28 \\
\hline 4으 & 3,40 & 0,28 & 1,41 \\
\hline \multicolumn{4}{|l|}{ Sensibilidade } \\
\hline 1으 & - & 94,60 & 83,80 \\
\hline $2 \underline{0}$ & - & 84,20 & 55,30 \\
\hline 3으 & - & 81,20 & 50,00 \\
\hline 4으 & - & 91,90 & 75,70 \\
\hline \multicolumn{4}{|l|}{ Coeficiente linear $\left(\beta_{0}\right)$} \\
\hline Com valores discrepantes & $-2,121$ & 4,582 & 53,002 \\
\hline Sem valores discrepantes & "não realizado" & 1,211 & $45,50^{2}$ \\
\hline \multicolumn{4}{|l|}{ Coeficiente angular $\left(\beta_{1}\right)$} \\
\hline Com valores discrepantes & 1,081 & 0,942 & $0,66^{2}$ \\
\hline Sem valores discrepantes & "não realizado" & 0,991 & 0,712 \\
\hline
\end{tabular}

ção, já que a hipótese dos parâmetros $\beta_{0}=0 \mathrm{e}$ $\beta_{1}=1$ foi aceita, indicando o ajuste dos dados pela equação da regressão (Tabela 1).

\section{Comparação da proporção de gestantes que sabem, ou não, referir seu peso anterior, peso final e estatura segundo características selecionadas}

Das 150 gestantes que referiram seu peso anterior, final e estatura, $96(64,0 \%)$ tinham idade entre 20 e 34 anos e 44 (29,3\%) tinham idade inferior a 19 anos.

De acordo com a Tabela 2, verifica-se que, entre as adolescentes, 38 (42,7\%) souberam referir as informações do peso anterior, final e estatura. Já entre as adultas, este percentual foi de $57,1 \%$.

Em relação ao grau de instrução, os dados evidenciaram que, das 113 gestantes com ensino fundamental completo ou mais, $72(63,7 \%)$ encontravam-se entre aquelas que souberam referir as três informações. Para o total de gestantes analfabetas ou com escolaridade até a quarta série $(n=72)$, observou-se que somente 


\section{Figura 1}

Diferenças entre as informações de peso final medido e referido, segundo as médias de peso final em gestantes no Município do Rio de Janeiro, Brasil.

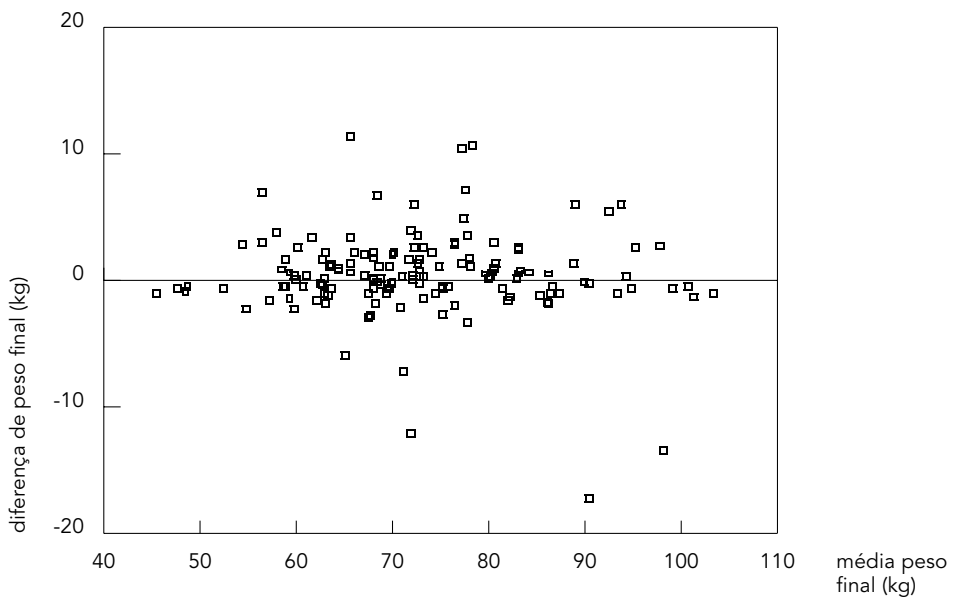

Figura 2

Diferenças entre as informações de estatura medida e referida, segundo as médias de estatura em gestantes no Município do Rio de Janeiro, Brasil.

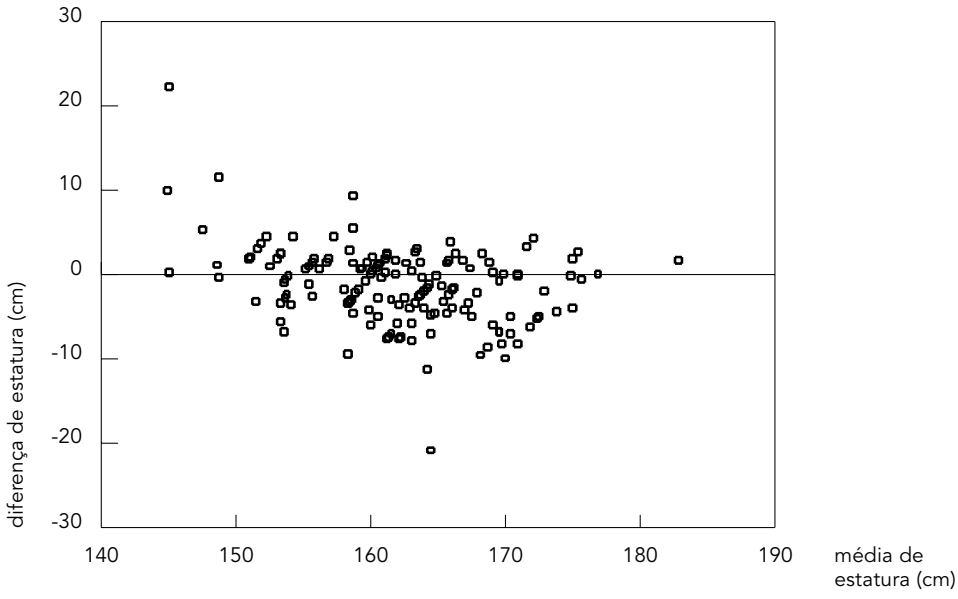

$26(36,1 \%)$ souberam referir seu peso anterior/ final e estatura.

Verificou-se um percentual considerável de gestantes $(59,3 \%)$ que soube referir o peso anterior, final e estatura entre as que iniciaram o pré-natal no primeiro trimestre de gestação. Para as que iniciaram a partir do segundo trimestre de gestação, somente $44(40,7 \%)$ souberam referir as três informações.

Pode-se observar que, entre as gestantes com mais de quatro consultas no pré-natal, $129(56,6 \%)$ souberam referir seu peso anterior, final e estatura. Para as que apresentaram menos de três consultas durante o período gestacional $(n=56)$, verificou-se que um percentual considerável $(64,3 \%)$ não soube referir as três informações.

Quanto à freqüência de pesagem nas consultas de pré-natal, 135 (54,4\%) das gestantes que sempre foram pesadas referiram adequadamente seu peso anterior, final e estatura. Cabe ressaltar que, das gestantes que nunca/às vezes foram pesadas, $28(65,1 \%)$ não souberam referir as três informações.

Em todas as variáveis analisadas, verificamse diferenças estatisticamente significativas no nível de $5 \%$.

\section{Discussão e considerações finais}

Vários métodos têm sido propostos para a validação das informações de peso e estatura 7,11, 23,24,25,26,27,28. O emprego simultâneo de vários métodos nesta pesquisa, permitiu-nos evidenciar o grau de importância e sensibilidade de cada um dos mesmos.

Destaca-se que as médias das diferenças medidas e referidas foram relativamente pequenas e estatisticamente não significativas no nível de 5\%. Devem ser lembradas as limitações referentes à utilização da média, já que valores discrepantes da distribuição não são levados em consideração. Vários trabalhos encontraram elevada correlação entre as informações medidas e as referidas quando avaliadas por meio do coeficiente de correlação de Pearson. Contudo, evidenciaram que as diferenças médias entre os valores medidos e referidos foram estatisticamente significativas 25,27,29.

Em virtude das limitações já referidas do coeficiente de Pearson como teste para avaliar a concordância 21,22 , acrescentou-se o uso do coeficiente de correlação intraclasse (CCIC), que leva em consideração o viés sistemático. Os valores encontrados para o CCIC do peso anterior, peso final e estatura foram elevados e concordantes com o trabalho de Chór et al. 11. 
Apesar de ambos os métodos, correlação de Pearson e CCIC, terem apontado elevada concordância/correlação entre as variáveis medidas e referidas, a análise de regressão linear foi mais sensível e capaz de detectar que essa relação é problemática para a variável estatura. Mesmo recorrendo ao método de exclusão das observações outliers, as quais poderiam estar distorcendo os dados analisados, mantiveramse os resultados significativos para os parâmetros avaliados $\beta_{0}$ e $\beta_{1}$, indicando a necessidade de avaliação diferenciada para esta variável, utilizando a equação da regressão como forma de ajuste para a determinação dos valores reais.

Com o método gráfico proposto por Bland \& Altman 21, realizou-se análise mais detalhada para investigar se as diferenças entre as informações medidas e referidas estavam relacionadas à média desses valores (informação referida somada à medida, dividindo-se por dois). Este método mostrou-se sensível, demonstrando haver tendência para subestimação do peso final e superestimação da estatura, independente dos valores de peso e estatura para essas gestantes. Tais achados foram discordantes de outros estudos que empregaram essa metodologia, como os de Gunnell et al. 25, Klag et al. 30, Nakamura et al. 27 e Olivarius et al. 28. Esses autores verificaram tendência de maior superestimação da estatura entre os "mais baixos" e maior subestimação do peso entre os "mais pesados".

Na utilização desse método gráfico para o peso pré-gestacional, evidenciou-se que as gestantes abaixo de $60 \mathrm{~kg}$ revelaram as menores diferenças médias, enquanto aquelas acima desse ponto apresentaram as maiores diferenças. Os valores foram bastante dispersos em relação à faixa média. Este fato pode ser explicado tanto em razão do pequeno tamanho amostral ( $n=30$ ), quanto em virtude das diferenças no ganho de peso relativas ao mês de entrada no pré-natal, ou seja, as gestantes que iniciaram o pré-natal no terceiro mês de gravidez possivelmente já tinham algum ganho de peso em comparação com aquelas que iniciaram no primeiro mês, conduzindo a maiores erros no cálculo das diferenças médias entre as informações medidas e referidas.

A análise de sensibilidade para as informações de peso e estatura medidos e referidos foi mais elevada no primeiro e último quartis. Resultados semelhantes foram encontrados por Boström \& Diderichsen 24. Os valores encontrados para o índice Kappa $(0,84$ para o peso e 0,55 para estatura) nessas distribuições foram considerados, segundo Fleiss 20, concordâncias de excelente a boa. Um possível fator associado com o diferencial na qualidade da informa-
Figura 3

Diferenças entre as informações de peso anterior medido e referido, segundo as médias de peso anterior em gestantes no Município do Rio de Janeiro, Brasil.

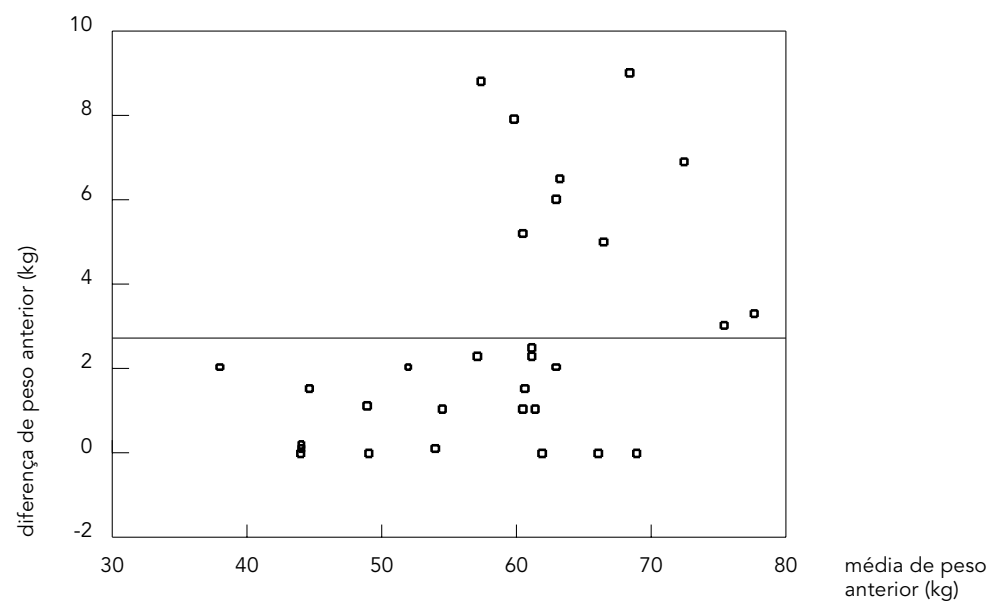

ção referida de peso e estatura entre os quartis, principalmente entre aquelas "mais leves/baixas" e "mais pesadas/altas", poderia ser o fato de essas gestantes serem consideradas de risco nutricional e, por isso, terem sido monitoradas mais freqüentemente durante o pré-natal e, assim, mais valorizada a informação.

Os achados deste trabalho devem ser interpretados no contexto de algumas limitações. $\mathrm{O}$ cálculo do tamanho amostral baseou-se no objetivo principal do estudo, que foi o de estimar as diferenças médias entre os pares dos valores medidos e referidos com nível de significância de $95 \%$ (totalizando um número de 150 gestantes estudadas). Ainda com relação à limitação do tamanho amostral, não era esperada uma perda de 120 gestantes para as análises quanto ao peso pré-gestacional. Somente trinta tiveram, no cartão de pré-natal, o registro do peso na primeira consulta (considerado como peso medido) e a semana gestacional, reduzindo significativamente a amostra e, conseqüentemente, o poder dos testes, gerando estatísticas com amplos intervalos de confiança.

Essa perda também esteve associada ao fato de, em alguns casos, a informação do peso na primeira consulta não estar sendo preenchida no cartão de pré-natal, impossibilitando a validação desta informação. É fundamental a conscientização de toda a equipe de saúde para a importância da medição do peso na primeira consulta pré-natal e o seu adequado pre- 
Comparação de algumas variáveis entre as gestantes hospitalizadas no Município do Rio de Janeiro que informaram, ou não, peso anterior/final e estatura.

\begin{tabular}{|c|c|c|c|c|c|}
\hline \multirow[t]{3}{*}{ Variáveis } & \multicolumn{4}{|c|}{ Informam 1} & \multirow[t]{3}{*}{$\mathrm{p}$} \\
\hline & \multicolumn{2}{|c|}{ Não } & \multicolumn{2}{|c|}{ Sim } & \\
\hline & $\mathrm{n}$ & $\%$ & $\mathrm{n}$ & $\%$ & \\
\hline \multicolumn{6}{|l|}{ Idade 1} \\
\hline Adolescentes & 51 & 57,3 & 38 & 42,7 & 0,02 \\
\hline Adultas & 84 & 42,9 & 112 & 57,1 & \\
\hline \multicolumn{6}{|l|}{ Escolaridade 1} \\
\hline Analfabeto + até $4 \underline{a}$ série & 46 & 63,9 & 26 & 36,1 & \\
\hline Ensino fundamental incompleto & 53 & 51,0 & 51 & 49,0 & 0,00 \\
\hline Ensino fundamental completo e + & 41 & 36,3 & 72 & 63,7 & \\
\hline \multicolumn{6}{|l|}{ Início do pré-natal1 } \\
\hline 1ㅇ trimestre & 72 & 40,7 & 105 & 59,3 & 0,00 \\
\hline 2ㅇ/3o trimestre & 64 & 59,3 & 44 & 40,7 & \\
\hline \multicolumn{6}{|l|}{ Número de consultas 1} \\
\hline $0-3$ & 36 & 64,3 & 20 & 35,7 & 0,00 \\
\hline $4 e+$ & 99 & 43,4 & 129 & 56,6 & \\
\hline \multicolumn{6}{|l|}{ Freqüência pesagem } \\
\hline Sempre2 2 & 113 & 45,6 & 135 & 54,4 & 0,01 \\
\hline Às vezes/nunca & 28 & 65,1 & 15 & 34,4 & \\
\hline Total gestantes avaliadas & 141 & 100,0 & 150 & 100,0 & \\
\hline
\end{tabular}

1 As variáveis início do pré-natal, número consultas, idade, escolaridade (entre as que informaram ou não peso anterior/final e estatura) não somam 100\%.

2 Gestantes que sempre foram pesadas durante o pré-natal.

enchimento no cartão de pré-natal, já que essa variável tem sido citada como uma das mais importantes na avaliação do ganho de peso na gestação e na prevenção de efeitos adversos fetais.

Uma outra questão refere-se à não-utilização de técnica adequada na aferição do peso prégestacional nas unidades de saúde. As discordâncias observadas entre os valores medidos e referidos para o peso anterior podem ter ocorrido por causa de erros na aferição dessa medida.

A possibilidade de avaliação do ganho de peso na gestação e suas conseqüências na saúde materno-fetal também foi afetada por essa perda, já que o $n$ amostral reduzido a trinta gestantes foi insuficiente para concluir sobre a utilização das informações referidas quanto ao peso pré-gestacional no "Estudo da Morbi-Mortalidade".

Excluindo aquelas que iniciaram o pré-natal no primeiro trimestre de gestação, verificou-se que o peso final foi mais facilmente reportado pelas gestantes. Possivelmente, aquelas que iniciaram o pré-natal tardiamente (já no segundo ou terceiro trimestre) possuíam, pelo menos, uma consulta ao final da gravidez em que o peso foi avaliado, contribuindo, dessa forma, para a concordância entre os valores medidos e referidos.

Já a estatura foi a pior informação referida pelas gestantes. $\mathrm{O}$ fato de ser uma medida pouco mensurada pelos profissionais nos serviços de saúde durante a realização do pré-natal, ao contrário da medida de peso, que é mais freqüentemente monitorada durante as visitas, explica, provavelmente, o elevado percentual de gestantes que não souberam referir sua estatura. Essa medição pelo menos uma vez durante a realização do pré-natal seria suficiente para garantir a informação correta por parte delas.

Outros estudos concernentes à validade das informações de estatura referidas durante a gravidez seriam necessários para a confirmação dos resultados aqui apresentados.

O grupo de gestantes que não informaram as três medidas foi o de menor nível de instrução, mais jovens, as que não fizeram ou menos 
freqüentaram o pré-natal e as que deram entrada mais tardiamente, já no segundo-terceiro trimestre de gestação, o que contribuiu para que fossem pesadas menos freqüentemente. Seria justamente nestas gestantes "desprivilegiadas” na assistência pré-natal que efeitos adversos/negativos estariam mais presentes. Portanto, pode-se inferir que a ausência das informações referidas é um marcador da qualidade de assistência pré-natal e de condições de vida. Esse fato é de grande importância, uma vez que tal grupo é mais vulnerável e necessitaria de maior atenção para conhecimento de sua situação de saúde e do planejamento relativo a ações que contemplassem melhores condições de vida. Desse modo, é preciso que os serviços de saúde no pré-natal desenvolvam estratégias para a captação/avaliação precoce destas gestantes como forma de prevenção de efeitos negativos.

\section{Resumo}

Os objetivos principais deste artigo são a avaliação da concordância entre as informações de peso e estatura medidas e referidas, bem como a identificação das principais características de 150 gestantes que souberam ou não referir seu peso anterior, peso final e estatura. Os resultados evidenciam que as informações referidas para o peso anterior aproximam-se dos valores medidos (coeficiente de correlação de Pearson $(0,96) e$ intraclasse $(0,92) ; p<0,05)$, o mesmo ocorrendo em relação à variável peso final (coeficiente de correlação de Pearson $(0,96)$ e intraclasse $(0,95) ; p<0,05)$. Contudo, a análise de regressão linear é capaz de detectar que essa relação é problemática para a variável estatura $\left(\beta_{0}=45,5\right.$ e $\left.\beta_{1}=0,71 ; p<0,05\right) e$, portanto, as informações referidas para esta devem ser utilizadas com cautela quando os valores medidos não estiverem disponíveis. Quanto à caracterização do grupo de gestantes que não informaram as três medidas $(n=141)$, elas são as de menor nivel de instrução (63,9\%), as que não fizeram ou menos freqüentaram o pré-natal (64,3\%) e as que deram entrada no pré-natal mais tardiamente - já no segundo ou terceiro trimestre (59,3\%).

Validade; Peso Corporal; Estatura; Gravidez
Nossos dados sugeriram que as informações referidas para o peso pré-gestacional aproximaram-se dos valores medidos, o mesmo ocorrendo em relação à variável peso final. Entretanto, para estatura, as informações referidas devem ser utilizadas com cautela quando os valores medidos não estiverem disponíveis.

Esforços a fim de conscientizar a população e os profissionais de saúde quanto à importância e à necessidade de implementação dessas medidas como rotina no acompanhamento durante o pré-natal certamente contribuiriam para a melhoria da qualidade dessas informações.

Como este trabalho avaliou apenas as gestantes que deram entrada em maternidades públicas, possivelmente diferentes resultados seriam obtidos se esta metodologia de validação fosse aplicada a gestantes que fizeram parte das maternidades privadas, onde esperaríamos encontrar um melhor nível sócio-econômico e de instrução.

\section{Colaboradores}

A. F. Oliveira participou da coleta de informações, estabeleceu o método do estudo e redigiu o artigo. A. M. J. Gadelha contribuiu na definição do desenho de estudo, na seleção de indicadores, discussão dos resultados e redação do artigo. M. C. Leal foi responsável pela revisão do artigo, contribuiu na definição do objeto de estudo, metodologia e discussão os resultados. C. L. Szwarcwald definiu a amostra, e análise estatística, colaborou na redação do artigo.

\section{Agradecimentos}

A Coordenação de Aperfeiçoamento de Pessoal de Nível Superior pelo apoio financeiro, e a Dra Vera Chiara (Professora do Departamento de Nutrição Social, Universidade do Estado do Rio de Janeiro) pela leitura crítica e apresentação de sugestões. 


\section{Referências}

1. Nucci LB, Duncan BB, Mengue SS, Branchtein L, Schmidt MI, Fleck ET. Assessment of weight gain during pregnancy in general prenatal care services in Brazil. Cad Saúde Pública 2001; 17:136774.

2. Krasovec K, Anderson M. Maternal nutrition and pregnancy outcomes: anthropometric assesssment. Scientific Publication 529. Washington, DC: Pan American Health Organization/World Health Organization; 1991.

3. World Health Organization. Maternal anthropometry for prediction of pregnancy outcomes: Memorandum from USAID/WHO/PAHO Mother Care Meeting. Bull World Health Organ 1991; 69:523-32.

4. World Health Organization. Maternal anthropometry and pregnancy outcomes: a WHO collaborative study. Bull World Health Organ 1995; 73 Suppl:1-98.

5. World Health Organization. Physical status: the use and interpretation of anthropometry. WHO Technical Report Series 854. Geneva: World Health Organization; 1995.

6. Niedhammer I, Bugel I, Bonenfant S, Goldberg M, Leclere A. Validity of self-reported weight and height in the French GAZEL cohort. Int J Obes 2000; 24:1111-8.

7. Ramalle-Gómara E, Mosquera-Lozano D, BreaHernando A, Gómez-Alamillo C. Validez de las medidas autodeclaradas de peso y talla en la estimación de la prevalencia de obesidad. Med Clin (Barc) 1997; 108:716.

8. Roberts RJ. Can self-reported data accurately describe the prevalence of overweight? Public Health 1995; 109:275-84.

9. Ziebland S, Thorogood M, Fuller A, Muir J. Desire for the body normal: Body image and discrepancies between self reported and measured height and weight in a British population. J Epidemiol Community Health 1996; 50:105-6.

10. Schmidt MI, Duncan BB, Tavares M, Polanczyk CA, Pellanda L, Zimmer PM. Validity of self-reported weight - A study of urban Brazilian adults. Rev Saúde Pública 1993; 27:271-6.

11. Chor D, Coutinho ESF, Laurenti R. 1999. Reliability of self-reported weight and height among state bank employees. Rev Saúde Pública 1999; 33:1623.

12. Lederman AS, Paxton A. Maternal reporting of prepregnancy weight and birth outcome: consistency and completeness compared with the clinical record. Matern Child Health J 1998; 2:123-6.

13. Schieve LA, Perry GS, Cogswell ME, Scanlon KS, Rosenberg D, Carmichael S, et al. Validity of selfreported pregnancy delivery weight: an analysis of the 1988 National Maternal and Infant Health Survey. Am J Epidemiol 1999; 150:947-56.

14. Stevens-Simon C, Roghmann KJ, McAnarney ER. 1992. Relationship of self-reported prepregnant weight and weight gain during pregnancy to maternal body habitus and age. J Am Diet Assoc 1992; 92:85-7.
15. Tomeo CA, Rich-Edwards JW, Michels KB, Berkey CS, Hunter DJ, Frazier AL, et al. Reproducibility and validity of maternal recall of pregnancy-related events. Epidemiology 1999; 10:774-7.

16. Yu SM, Nagey DA. Validity of self-reported pregravid weight. Ann Epidemiol 1992; 2:715-21.

17. Leal MC, Gama SGN, Campos MR, Cavalini LT, Garbayo LS, Brasil C, et al. Estudo da morbi-mortalidade e da atenção peri e neonatal no Município do Rio de Janeiro. Cad Saúde Pública; no prelo.

18. Zar JH. Biostatistical analysis. New Jersey: Prentice Hall Direct; 1998.

19. Lhoman TG, Roche AL, Martorell R. Anthropometric standardization reference manual. Champaign: Human Kinetics Books; 1988.

20. Fleiss JL. Statistical methods for rates and proportions. New York: John Wiley \& Songs; 1981.

21. Bland JM, Altman DG. Statistical methods for assessing agreement between two methods of clinical measurement. Lancet 1986; 8:307-10.

22. Kramer MS, Feinstein AR. Clinical biostatistics: The biostatistics of concordance. Clin Pharmacol Ther 1981; 29:111-23.

23. Bolton-Smith C, Woodward M, Tunstall-Pedoe H, Morrison C. Accuracy of the estimated prevalence of obesity from self reported height and weight in an adult Scottish population. J Epidemiol Community Health 2000; 54:143-8.

24. Boström G, Diderichsen F. Socioeconomic differentials in misclassification of height, weight and body mass index based on questionnaire data. Int J Epidemiol 1997; 26:860-6.

25. Gunnell D, Berney L, Holland P, Maynard M, Blane D, Frankel S, et al. How accurately are height, weight and leg length reported by the elderly, and how closely are they related to measurements recorded in childhood? Int J Epidemiology 2000; 29:456-64.

26. Jeffery RW. Bias in reported body weight as a function of education, occupation, health and weight concern. Addict Behav 1996; 21:217-22.

27. Nakamura K, Hoshino Y, Kodama K, Yamamoto M. Reliability of self-reported body height and weight of adult Japonese women. J Biosoc Sci 1999; 31:555-8.

28. Olivarius NF, Andreasen AH, Leken J. Accuracy of 1-, 5- and 10-year body weight recall given in a standard questionnaire. Int J Obes 1997; 21:67-71.

29. Norgan NG, Cameron N. The accuracy of body weight and height recall in middle-aged men. Int J Obes 2000; 24:1695-8.

30. Klag MJ, He J, Mead LA, Ford DE, Pearson TA, Levine DM. Validity of physicians' self-reports of cardiovascular disease risk factors. Ann Epidemiol 1993; 3:442-7.

Recebido em 3/Jan/2002

Versão final reapresentada em 14/Out/2002

Aprovado em 18/Jun/2003 